\title{
Persuasive Technology for Human Well-Being: Setting the Scene
}

\author{
Wijnand IJsselsteijn, Yvonne de Kort, Cees Midden, Berry Eggen, \\ and Elise van den Hoven \\ Eindhoven University of Technology, \\ P.O.Box 513, $5600 \mathrm{MB}$, Eindhoven, The Netherlands \\ \{w.a.ijsselsteijn, y.a.w.d.kort, c.j.h.midden, \\ j.h.eggen, e.v.d.hoven\}@tue.nl
}

\begin{abstract}
In this short paper we aim to give a brief introduction to persuasive technology, especially as it pertains to human well-being. We discuss a number of current research opportunities in areas of healthcare, environmental conservation, and education. We conclude by highlighting what we regard as the key research challenges that need to be addressed, focusing on context sensing and appropriate feedback, the need for longitudinal user studies, and ethical concerns.
\end{abstract}

\section{Introduction}

Persuasion is part and parcel of human interaction. From the serpent in the Garden of Eden to our modern mass-media society, persuasive efforts abound in a continuous attempt to influence our attitudes and behaviours, convincing us to spend money on one product rather than another, to vote for a particular political party, to stop smoking, to exercise more, to fight for environmental conservation, animal wellbeing, better schools. Or to eat an apple.

Since long, media technology has played a significant role in facilitating the delivery of persuasive messages to purchase, donate, vote, concede, or act - from megaphones to billboards to television. Technology becomes an especially powerful tool when it allows the persuasive techniques to be interactive rather than one-way, that is, altering and adjusting the pattern of interaction based on the characteristics or actions of the persuaded party - the user's inputs, needs, and context. This realisation has led to the investigation of persuasive technology [1,2], defined as a class of technologies that are intentionally designed to change a person's attitude or behaviour. Importantly, persuasion implies a voluntary change of behaviour or attitude or both. If force (coercion) or misinformation (deception) are used, these would fall outside of the realm of persuasive technology.

Humans are arguably the strongest persuaders. They have an unmistakable social presence and impact, can sense the appropriate timing, mood and context as opportunities for another person to be persuaded, and have a keen, mostly intuitive sense of the social psychological principles of persuasion, such as praise, reciprocation, similarity, or authority. However, as Fogg [2] points out, computers can 
have a number of distinct advantages over human persuaders. They can be more persistent (irritatingly so, in fact), they can allow anonymity (useful in cases where sensitive issues are at play), they can access and control a virtually unlimited store of data (retrieving exactly the right nuggets of information at the right time), and they can use many modalities (text, audio and video clips, rich graphics and animations, etc.) to create a seamless and convincing experience. Moreover, successful pieces of persuasive software can be easily replicated and distributed (addressing large numbers of people at the same time), and, with computers becoming increasingly ubiquitous and embedded, persuasive technology may gain access to areas where human persuaders would not be welcomed (e.g., bedroom, bathroom) or are physically unable to go (e.g., inside clothing or household appliances).

\section{Persuasive Technology for Human Well-Being}

Although the most frequent application of persuasive technology today is the use of computers to sell products and services, there is great beneficial potential in applying persuasive technologies to increase human health and well-being. The use of interactive technology in the health arena is still in its early stages, with e-care and tele-care programmes to extend healthcare into people's home environments not yet living up to their full potential. Arguably, one of the strongest areas of innovation for persuasive technology in the near future will be preventive health engineering.

Technological developments in ubiquitous computing and ambient intelligence allow for new opportunities in this area [3]. In particular, the development of new sensor technologies and algorithms that allow for context-aware computing, will make it possible to infer elements of a person's context and activity, and deliver appropriate persuasive health-related messages to that person at the right time when decisions are made or behaviour is executed, i.e., just-in-time messaging [4]. Moreover, the embedding of computational power and interactive displays in our everyday environment [5] as well as the ubiquity of mobile computing devices worn or carried by an increasing number of users, makes it feasible to provide persuasive feedback at the appropriate place where the user is likely to benefit most.

Technologies that motivate and support healthier lifestyle decisions related to diet, exercise, smoking, sexual behaviour, TV and internet use, stress management, and maintaining social relationships could delay or even prevent the onset of a variety of medical problems, and improve the quality of life. Potentially significant savings in healthcare costs, together with a shortage of medical and healthcare professionals, are likely drivers for insurance companies and healthcare providers to invest in this area. A number of early explorations are available, with more ambitious programmes currently being implemented (e.g., Intel, HP Labs, Philips). Examples include virtual fitness coaches for motivating workouts in healthy (though perhaps slightly overweight) adults $[6,7]$, QuitKey, a mobile application for helping people beat their smoking habit through cueing a gradual reduction programme [8], MyFoodPhone, which promotes healthy eating habits by keeping track of food intake and biometric data, and connecting to a nutrition coach who gives personalised feedback [9], or the recent use of social network visualisations as behavioural feedback displays, raising awareness of social connectedness as a dynamic and controllable aspect of well-being [10]. 
Furthermore, in the healthcare domain, a significant research effort is focused on gerontechnology, that is, technology in the service of healthy ageing. Motivated by trends in the world's demographics (i.e., increasing number of elderly people and increasing life spans), our basic need for autonomy, as well as constrained healthcare resources, technologies are investigated that will ensure the independence of the older adult and increase the quality of later life. Many of the challenges and problems of old age could be overcome by enduring life style or behaviour changes, such as dietary changes, exercise behaviour, or compliance to prescribed medication regimes. Other problems may be prevented through a continuation of already existing healthy behaviours, such as maintenance of an active social network and engaging in leisure activities. Persuasive technologies can have a positive, supportive role by convincing, stimulating or motivating adult users to engage in healthy behaviours, and instantly rewarding such behaviours when they occur [3, 11]. Paradoxically, in order to be effective, persuasive gerontechnology should perhaps not focus primarily on the older adult, but should start already much earlier in life, as trajectories leading to chronic diseases in later life (e.g., cardiovascular diseases, Alzheimer's disease) are severely influenced by one's lifestyle choices as a younger adult $[3,12]$.

In other areas of human well-being, such as education and environmental conservation, persuasive technology will also have a significant part to play. Here too, appropriate just-in-time notifications presenting relevant, actionable information in situ may prove to be very powerful tools in motivating people to adapt their behaviour. For example, direct feedback on energy consumption when turning the heater up, taking a shower or using a household appliance [13] can trigger a positive behaviour change. To raise environmental awareness and change energy use patterns, computer games such as PowerHouse [14] are also explored for their ability to provide information in a fun and rewarding way, creating an understanding of the relation between various domestic behaviours and household energy consumption. Given the huge success of computer games amongst children and adolescents, educators are currently looking at the opportunities for motivating learning experiences through the engaging interactivity and subtle reward structure of computer games, both inside and outside the classroom $[15,16]$.

\section{Three Key Research Challenges}

Although the first design explorations of persuasive technology show promising results, we would like to conclude this short paper by identifying three key research challenges that need to be addressed as persuasive technology matures. First, when applying context-sensing and inferences for just-in-time persuasive messaging, the benefits that such interventions will bring are crucially dependent on the quality and relevance of the machine sensing and inference algorithms. As the physical world and human behaviour are both highly complex and ambiguous, this is by no stretch of the imagination a solved problem. Most problematic will be attempts at inferring some internal human intent, requiring levels of intelligence even a human would find difficult to attain. 
Second, human behaviour typically takes a long time to change. At present, to our knowledge, hardly any user studies are available that have looked at the long-term effectiveness of persuasive technology. Will the technology be perceived as subtle and non-irritating, or as bullying or patronising? What happens when we receive the same feedback not once or twice, but continuously, day in day out? How tolerant will we be to incorrect inferences, improper feedback, or bad timing? Will we remain motivated after we have stopped using the persuasive technology, removing the external incentives that influenced our behaviour? These and related issues need to be addressed in longitudinal, large- $\mathrm{N}$ user acceptance studies.

And finally, there is the need for a continuous and open ethical debate on the pros and cons of persuasive technology. As technological progress enables more subtle and effective persuasive techniques to be implemented, this will continue to pose new moral dilemmas. User-centred design of persuasive technology should explicitly include ethical guidelines [17], encouraging morally responsible design of persuasive technology and, of course, frowning upon the serpent in the Garden.

\section{References}

1. Fogg, B.J.: Persuasive technologies. Communications of the ACM 42(5) (1999) 27-29

2. Fogg, B.J.: Persuasive Technology. Using Computers to Change What We Think and Do. San Fransisco, Morgan Kaufman Publishers (2003)

3. Intille, S.S.: A new research challenge: Persuasive technology to motivate healthy aging. IEEE Transactions on Information Technology in Biomedicine, 8 (2004) 235-237

4. Intille, S.S.: Designing a home of the future. IEEE Pervasive Computing 1, April/June (2002) 80-86

5. Aarts, E., Marzano, S.: The New Everyday. Views on Ambient Intelligence. Rotterdam, 010 Publishers (2003)

6. Davis, J.W., Bobick, A.F.: Virtual PAT: a Virtual Personal Aerobics Trainer. Proc. of Workshop on Perceptual User Interfaces (PUI'98), San Francisco, CA (1998) 13-18

7. IJsselsteijn, W.A., de Kort, Y.A.W., Westerink, J., de Jager, M., Bonants, R.: Fun and Sports: Enhancing the Home Fitness Experience. In: Rauterberg, M. (ed.), Entertainment Computing - ICEC 2004. LNCS, 3166, New York, Springer Verlag (2004) 46-56

8. http://www.quitkey.com/

9. http://www.myfoodphone.com/

10. Morris, M.E.: Social networks as health feedback displays. IEEE Internet Computing 9(5) (2005) 29-37

11. de Kort, Y.A.W., IJsselsteijn, W.A., Midden, C.J.H., Eggen, J.H., van den Hoven, E.A.W.H.: Persuasive gerontechnology. Gerontechnology 4 (2005) 123-127

12. Morris, M.E.: Biofeedback revisited: Dynamic displays to improve health trajectories. In: IJsselsteijn, W.A., de Kort, Y.A.W., Midden, C.J.H., Eggen, J.H., van den Hoven, E.A.W.H. (eds.), PERSUASIVE 2006. LNCS, 3962, New York, Springer Verlag (2006) 207-214

13. McCalley, T., Kaiser, F., Midden, C.J.H., Keser, M., Teunissen, M.: Persuasive appliances: Goal priming and behavioural response to product-integrated energy feedback. In: IJsselsteijn, W.A., de Kort, Y.A.W., Midden, C.J.H., Eggen, J.H., van den Hoven, E.A.W.H. (eds.), PERSUASIVE 2006. LNCS, 3962, New York, Springer Verlag (2006) 45-49 
14. Bang, M., Torstensson, C., Katzeff, C.: The PowerHouse: A persuasive computer game designed to raise awareness of domestic energy consumption. In: IJsselsteijn, W.A., de Kort, Y.A.W., Midden, C.J.H., Eggen, J.H., van den Hoven, E.A.W.H. (eds.), PERSUASIVE 2006. LNCS, 3962, New York, Springer Verlag (2006) 123-132

15. http://www.seriousgames.org/

16. Lucero, A., Zuloaga, R., Mota, S., Muñoz, F.: Persuasive technologies in education: Improving motivation to read and write for children. In: IJsselsteijn, W.A., de Kort, Y.A.W., Midden, C.J.H., Eggen, J.H., van den Hoven, E.A.W.H. (eds.), PERSUASIVE 2006. LNCS, 3962, New York, Springer Verlag (2006) 142-153

17. Berdichevsky, D., Neuenschwander, E.: Towards an ethics of persuasive technology. Communications of the ACM 42(5) (1999) 51-58 